\title{
Revista Facultad de Ciencias Básicas ISSN 1900-4699
}

\section{ANTECEDENTES Y PERSPECTIVAS PARA EL MANEJO INTEGRADO DE Tuta absoluta Meyrick 1917 (Lepidoptera: Gelechiidae)}

\author{
BACKGROUND AND PERSPECTIVES OF THE INTEGRATED \\ MANAGEMENT OF Tuta absoluta Meyrick 1917 \\ (Lepidoptera: Gelechiidae)
}

\author{
María HERRERA ROCHA', Daniel RODRÍGUEZ CAICEDO', Fernando CANTOR'* \\ ${ }^{1}$ Laboratorio Control Biológico, Universidad Militar Nueva Granada, kilómetro 2 vía Cajicá- \\ Zipaquirá, Cajicá, Colombia. \\ *Autor corresponsal. E-mail: control.biologico@unimilitar.edu.co
}

En los últimos años se han realizado diversos avances en términos de investigación para un manejo adecuado de Tuta absoluta, una de las principales plagas del tomate en el mundo. En los cultivos convencionales el método de control químico ha sido el más utilizado. Actualmente se buscan estrategias sostenibles para el manejo integrado de plagas (MIP). Apanteles gelechiidivoris, parasitoide especifico de Tuta absoluta encontrado de forma natural en Colombia, es considerado como una estrategia promisoria para su implementación en un programa de MIP del tomate. A pesar de esto, exposiciones a pesticidas pueden afectar su efectividad de control dentro del cultivo. La evaluación de los efectos de estos pesticidas sobre los enemigos naturales de Tuta absoluta permite anticipar sus perspectivas de éxito. Dentro de los ensayos realizados frecuentemente para determinar la compatibilidad se llevan a cabo evaluaciones de dosis letal media DL50, exposición directa y exposición indirecta. En la actualidad también se adelantan trabajos sobre aspectos que afectan la biología de los controladores más allá de la mortalidad inducida directamente sobre estos, como evaluaciones de fecundidad, emergencia de adultos, capacidad parasítica, longevidad y proporción sexual. En este trabajo se revisan los avances más significativos en el control de Tuta absoluta mediante el empleo de A. gelechiidivoris y se discuten aspectos relativos a la necesidad de adelantar estudios de compatibilidad del parasitoide con estrategias de manejo químico.

Palabras clave: MIP, cogollero del tomate, parasitoides, depredadores.

\section{Abstract}

In recent years, there have been advances in the research into proper management of Tuta absoluta, one of the major tomato pests in the world. In conventional crops, the chemical control method has been the most broadly used but more sustainable strategies for integrated pest management (IPM) are desirable. Apanteles gelechiidivoris, a specific parasitoid of Tuta absoluta naturally found in Colombia, is considered a promising strategy for its implementation in tomato IPM. Despite this, exposure to pesticides may affect its control effectiveness in crops. Assessing the effects of exposure to these pesticides on natural enemies of Tuta absoluta allows us to anticipate their prospects of success. Tests frequently performed to determine compatibility are lethal dose (LD50), direct and indirect exposure, among others. Currently, research has also been conducted into aspects affecting the biology of controllers beyond directly induced mortality such as fertility, adult emergence, parasitic capacity, longevity and sex ratio. This paper reviews the most significant advances in controlling Tuta absoluta using A. gelechiidivoris and discusses the need to carry out compatibility studies of this parasitoid with chemical management strategies. 


\section{INTRODUCCIÓN}

El tomate es una de las principales hortalizas cultivadas en Colombia. Su producción se hace de dos formas: a campo abierto y bajo condiciones confinadas de invernadero. El principal productor bajo invernadero del país en 2014 fue Boyacá, con 115686 toneladas, que representaron el 49,2\% de la producción total nacional (Agronet, 2016). Sin embargo, la producción de este cultivo está sujeta al ataque de diferentes plagas y enfermedades (Denez et al., 2014; Silva et al., 2016), entre otros factores, lo que provoca mayores costos en la producción, reducciones en la calidad y cantidad del fruto y disminución en el rendimiento.

El cogollero del tomate, Tuta absoluta Meyrick 1917 (Lepidoptera: Gelechiidae), es una de las plagas más comunes en los cultivos de tomate en Colombia (Morales et al., 2013, 2014). Las pérdidas causadas por esta plaga dependen del estado fenológico de la planta hospedera, pero alcanzan hasta $100 \%$, debido al ataque sobre hojas, flores, tallos y frutos (Guedes y Picanço, 2012; Martins et al., 2016). Para su control se usan frecuentemente productos químicos, especialmente de amplio espectro, como carbamatos, organofosforados y piretroides (Gul et al., 2016). Para el caso del control en T. absoluta, imidacloprid, spinosad, triflumurón, clorfenapir y abamectina son los más usados (Campos et al., 2014; Collavino y Giménez, 2001; Virgala, 2006). Sin embargo, la eficiencia de este tipo de control puede disminuir debido al hábito de desarrollo del cogollero del tomate, cuyo estado larval se lleva a cabo en tejidos internos de la planta, lo que le permite una cierta protección frente al efecto de insecticidas de contacto. Además, T. absoluta posee una alta habilidad de desarrollar resistencia a insecticidas como abamectina y spinosad, como ha sido reportado por numerosos autores (Campos et al., 2014; Ghoneim, 2014; Mohan et al., 2007; Siqueira et al., 2001; Vacas et al., 2013, entre otros).

Por situaciones como las planteadas anteriormente Bawin et al. (2014) hacen referencia a que las estrategias actuales de control deben buscar la reducción de los pesticidas sintéticos y la integración de diferentes estrategias.

Según Bajonero et al. (2008) en Colombia uno de los principales agentes biológicos para el control de T. absoluta es el parasitoide Apanteles gelechiidivoris Marsh 1975 (Hymenoptera: Braconidae), que ataca con preferencia larvas de tercer instar de T. absoluta como recurso de oviposición. Existen reportes de efectividad en campo de este método de control hasta del $80 \%$ cuando la estrategia es combinada con el control etológico (Morales et al., 2014).

Para que un cultivo comercial sea sostenible es necesario el uso combinado y racional de diferentes tácticas de control (Hardy, 2011), y es claro que el control químico sigue siendo una de las piedras angulares del MIP, especialmente en cultivos hortícolas (Abbes et al., 2015). Por lo tanto, el uso de pesticidas selectivos es fundamental para el éxito en el control de plagas o enfermedades, siempre y cuando cause los menores impactos sobre otros componentes del sistema, como los enemigos naturales. Por esta razón es necesario realizar estudios acerca de los efectos de los productos fitosanitarios sobre los agentes benéficos (Desneux et al., 2007). Estudios de selectividad constituyen el primer paso hacia la formulación de directrices sobre el uso de métodos apropiados de control (Babendreier et al., 2005).

Diversos estudios de selectividad de pesticidas con respecto a agentes de control biológico se han basado en la determinación de sus efectos letales mediante estudios de dosis letal media ( 2550$)$ o concentración letal (CL50) (Desneux et al., 2007; Sattar et al., 2011; Talebi et al., 2008; Umoru y Powell, 2002). Los estudios recientes tienen en cuenta efectos a nivel de poblaciones y efectos subletales (Castiglioni et al., 2008). A pesar de esto no existen suficientes investigaciones acerca de los efectos letales y subletales de productos fitosanitarios sobre especies no objetivo (Santos et al., 2016) y particularmente los efectos subletales han sido menos estudiados (Desneux et al., 2007; Garcia, 2009). Estos últimos pueden ser definidos como efectos sobre la fisiología o el comportamiento de los individuos que sobreviven a la exposición de los pesticidas (Desneux et al., 2007).

\section{RESULTADOS Y DISCUSIÓN}

\section{SITUACIÓN DE Tuta absoluta EN LOS CULTIVOS DE TOMATE}

Originaria de Sudamérica, el primer reporte de T. absoluta (Lepidoptera: Gelechiidae) ocurrió en Perú en 1917 (Razuri y Vargas 1975; Vargas, 1970). Con el tiempo esta plaga se propagó a lo largo de los países europeos y africanos productores de tomate (Desneux et al., 2010; Roda et al., 2015).

De acuerdo con la revisión realizada por Tonnang et al. (2015), fuera de su área de origen por primera vez se reporta la presencia de T. absoluta en España en 2006, desde donde se cree que se extendió a otros países como Italia (2008), Francia (2008), Albania (2009), Bulgaria (2009), Portugal (2009), Holanda (2009), Reino Unido (2009) y Serbia (2011); así como a la región del Medio Oriente: Israel (2010), Irán (2010) y Turquía (2010). Posteriormente alcanzó las Islas Canarias en 2012. En el norte de África T. absoluta fue encontrada en Argelia (2008), Marruecos (2008), Egipto (2009), Libia (2009) y Túnez (2009) (Fig. 1). En el África subsahariana en 2012 se registran reportes en Etiopía, Nigeria, Senegal, Sudán, y más recientemente en Tanzania, Uganda y Kenia. Las detecciones en Kenia fueron confirmadas tanto por el uso de caracteres morfológicos como por técnicas moleculares (DNA barcoding). Nuevos hallazgos de T. absoluta fueron reportados por Shashank et al. (2015) en India y por Ajaya et al. (2016) en Nepal, los cuales se deben sumar a la revisión de Tonnang et al. (2015). 
Figura 1. Mapa de distribución mundial de T. absoluta (EPPO Global Database, 2017).

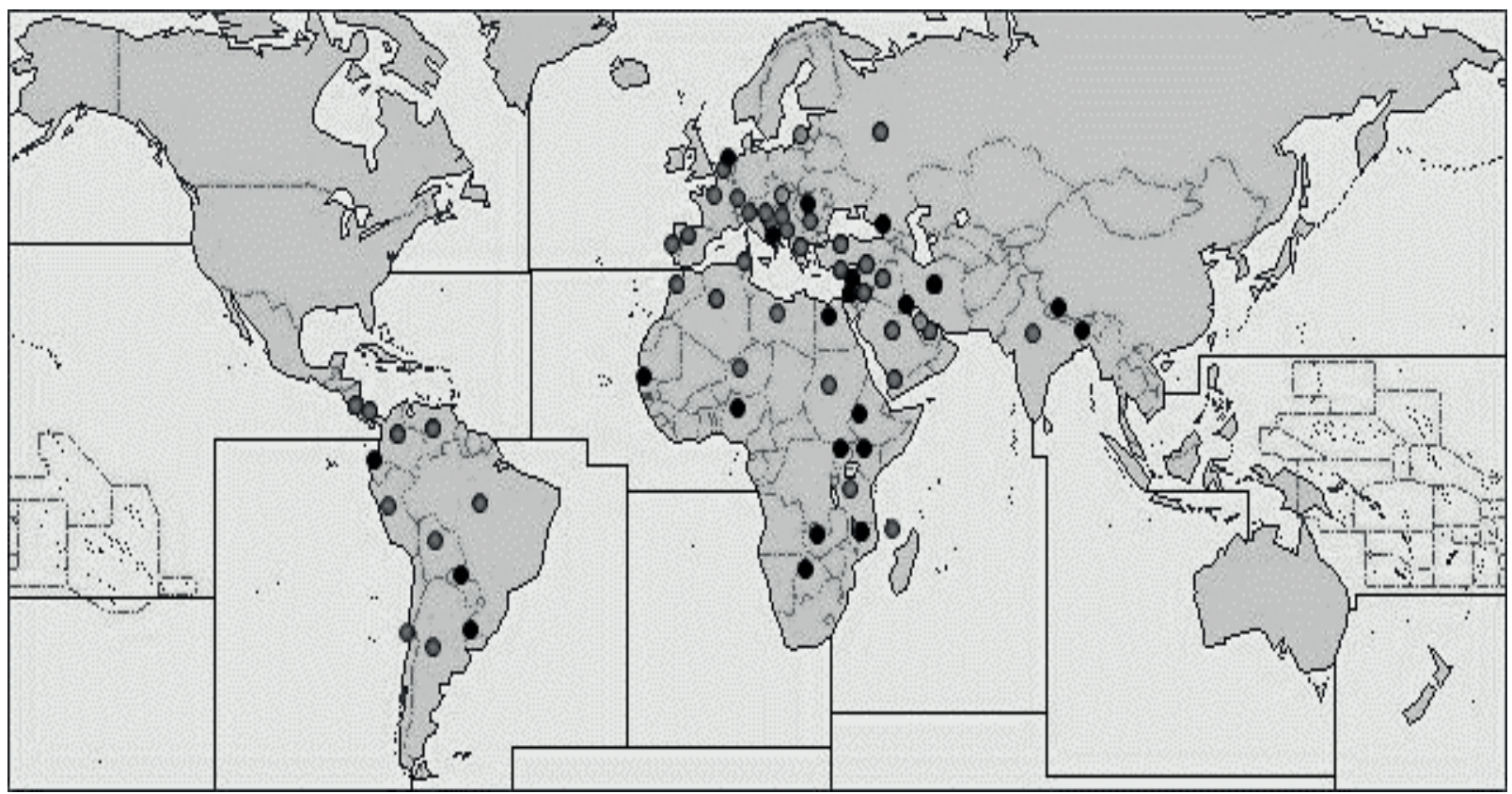

T. absoluta es una de los principales responsables de la reducción en la producción de tomate en Colombia (GómezValderrama et al., 2014) debido a su gran capacidad para desarrollarse y su rápida difusión a nuevas áreas causando daños económicamente relevantes (Grousset et al., 2015). Las larvas de este lepidóptero presentan cuatro estadios que se desarrollan dentro de galerías en las hojas, alimentándose del mesófilo y dejando la epidermis intacta. Producen la expansión de las galerías, lo que genera una pérdida de la actividad fotosintética de la planta (Cocco et al., 2014). Con frecuencia las larvas salen de la hoja para iniciar nuevas galerías o desplazarse a otras hojas o frutos (Monserrat, 2009; Tropea Garzia et al., 2012; Bawin et al., 2014).

\section{BIOECOLOGÍA DE T. absoluta}

El ciclo de vida consta de cuatro estados de desarrollo: huevo, larva, pupa y adulto. Las hembras de esta plaga ovipositan frecuentemente sobre las hojas (Urbaneja et al., 2013). Los huevos son ovalados y de superficie lisa, de color blanco perlado inicialmente y se van tornando amarillos conforme se desarrollan. Según Vélez (1997), el tórax y el abdomen de larvas de primer instar son de color crema y su cabeza es negra. En el cuarto instar aparece una coloración roja en el dorso que se extiende longitudinalmente desde los ocelos hasta la parte posterior del cuerpo (Urbaneja et al., 2013). Los individuos pupan principalmente en las hojas y en el suelo, y ocasionalmente en los vástagos laterales y en los frutos. Una vez formada, la pupa presenta una coloración verde que posteriormente se torna marrón (Medeiros, 2007). En condiciones controladas de laboratorio de $25 \pm 2{ }^{\circ} \mathrm{C}, 60 \pm 10 \%$ $\mathrm{HR}$, las pupas tienen una duración de siete a diez días. Después de la emergencia cada hembra puede depositar de 55 a 130 huevos, que eclosionan entre tres y siete días después. Existe evidencia de que en condiciones de invernadero la mayor concentración de posturas se encuentra en el tercio superior de las plantas y la mayoría de los huevos son depositados en las hojas (Medeiros et al., 2009).

\section{PREVENCIÓN Y CONTROL DE T. absoluta}

El manejo del cogollero del tomate puede ser realizado de diferentes maneras tanto preventivas como correctivas. Un programa de manejo integrado de plagas (MIP) enfatiza en la combinación de métodos, tal como el control químico y control biológico, para mantener las poblaciones por debajo del umbral económico. A través de la combinación de métodos se logra a largo plazo un manejo más sostenible de la plaga que el que resulta del uso de un solo método (Roubos et al., 2014). En Colombia el método de control más utilizado es el químico, en dosis y frecuencias elevadas (Vallejo, 1999; Jaramillo et al., 2013).

\section{CONTROL BIOLÓGICO DE T. absoluta}

\section{Entomopatógenos}

Una alternativa escasamente estudiada para el control de T. absoluta es el uso de entomopatógenos, entre los cuales se encuentran hongos, bacterias, nematodos y virus.

Según la revisión de Gómez (2014), los hongos entomopatógenos Beauveria bassiana y Metarhizium anisopliae, producen en condiciones de laboratorio mortalidades en larvas de T. absoluta que oscilan entre el $30 \%$

\section{Revista FULTAD DE CIENCIAS BÅSICAS}


y el 86 \% (Giustolin et al., 2001; Kaoud, 2014; Rodríguez et al., 2006; Torres Gregorio et al., 2009).

Rodríguez et al. (2006) concluyeron que el hongo entomopatógeno B. bassiana presenta control entre $60 \%$ y 90 \% según la condición de exposición. También fue evaluado M. anisopliae var. Anisopliae, con el cual se presentó una menor mortalidad de las larvas con respecto a B. bassiana. Sin embargo, cuando huevos de T. absoluta eran expuestos a $M$. anisopliae, el control alcanzó el 80 \% (Pires et al., 2010). Otro de los organismos entomopatógenos usados frecuentemente es la bacteria Bacillus thuringensis. Estudios de efectividad de esta bacteria muestran una mortalidad de larvas entre $80 \%$ y 100 \% (Ramírez et al., 2010). Sin embargo, estos resultados no han sido consistentes en condiciones más reales, como en invernadero (Delbene, 2003).

Según González-Cabrera et al. (2010) y Luna et al. (2012), el uso de B. thuringiensis para el control de T. absoluta es frecuente entre los productores de tomate como estrategia de manejo sostenible.

En relación con el uso de nematodos, Batalla-Carrera et al. (2010) demostraron que pueden ser eficaces: Steinernema feltiae, S. carpocapsae y Heterorhabditis bacteriophora en invernadero controlaron larvas entre un $87 \%$ y un $95 \%$.

En Colombia se han realizado estudios orientados a encontrar y evaluar la presencia de granulovirus aislados de T. absoluta en Boyacá y Cundinamarca. Gómez-Valderrama et al. (2014) obtuvieron ocho aislados provenientes de larvas infectadas, con potencial de control de la plaga en cultivos de tomate.

\section{CONTROL ETOLÓGICO}

La comunicación química también ha sido estudiada como alternativa al uso de productos químicos (González et al., 2012), y el uso de feromonas se considera la primera línea de defensa contra T. absoluta tanto en campo abierto como en invernaderos, ya que se utilizan para fines de seguimiento y eliminación de machos (Lobos et al., 2013; Megido et al., 2013). Esta técnica está patentada por la empresa ShinEtsu Chemical Co. Ltd. de Tokyo (Mochizuki et al., 2012). El principal componente de la feromona es (3E, 8Z, 11Z)-3,8,11tetradecatrien-1-yl acetato, identificado por Attygalle et al. (1995), citados en Mafra-Neto et al. (2010). Además del monitoreo, las feromonas han sido empleadas con éxito para el control de adultos de T. absoluta, para detección temprana (Van der Straten et al., 2011) y para la técnica de interrupción de la cópula (Vacas et al., 2011; Chermiti y Abbes, 2012; Caparros et al., 2013; Cocco et al., 2013). Tal es el caso en Italia, donde empleando una densidad de mil dispensadores por hectárea se obtuvo una reducción entre el $93 \%$ y el $99 \%$ del daño en hojas y frutos (Cocco et al., 2013). De acuerdo con este estudio actualmente esta estrategia puede ser más costosa que el control químico convencional. Sin embargo, los autores sugieren que pueden reducirse costos si se optimizan las tasas de emisión del producto.
Algunos autores, como Michereff Filho et al. (2000), Hassan y Al-Zaidi (2009), Vacas et al. $(2011,2013)$ y más recientemente Taha et al. (2013), han expresado que estrategias basadas en el uso de feromonas para el manejo de T. absoluta muestran pocos resultados, aparentemente por la habilidad de las hembras de reproducirse por partenogénesis deuterotóquica (Caparros et al., 2012). Por lo anterior, estas estrategias deben ser implementadas dentro de programas MIP haciendo uso de distintos tipos de trampas, tales como las delta, las de agua o las ubicadas en zonas de cargue y descargue de camiones, tal como lo mencionan Van der Straten et al. (2011) para el caso de Holanda.

En Colombia Morales et al. (2014) realizaron estudios de acción combinada entre el parasitoide A. gelechiidivoris y una feromona cuyo componente principal es tetradicatrienelina ( $3 E, 8 Z, 11 Z)$. Una de las conclusiones obtenidas de este trabajo es que el conocimiento de la distribución espacial de la plaga permite tomar medidas de control sobre el cultivo desde sus etapas iniciales. Es decir, medidas como la instalación de trampas de feromona y la liberación de avispas de $A$. gelechiidivoris aseguran un control constante en el tiempo. De esta manera se logra una efectividad en el control de poblaciones de la plaga de hasta un $86,38 \%$.

\section{CONTROL BIOLÓGICO CON ENTOMÓFAGOS}

Según Van der Straten et al. (2011), investigaciones en España evidencian que el chinche depredador Macrolophus pygmaeus Rambur (Heteroptera: Miridae) puede controlar muy bien tanto poblaciones de moscas blancas como de T. absoluta. El chinche consume huevos y pequeñas larvas de T. absoluta, pero sus poblaciones se desarrollan lentamente, razón por la cual es necesario realizar múltiples introducciones adicionales (Urbaneja et al., 2009). Sin embargo, en invernaderos holandeses no se evidencian efectos de Macrolophus pygmaeus (Hemiptera: Miridae) sobre T. absoluta cuando es baja la densidad de población de la plaga. Para los mismos autores (Van der Straten et al., 2011) altas infestaciones de T. absoluta en el suroriente de Europa fueron el sustrato para el desarrollo de poblaciones de parasitoides nativos (Desneux et al., 2010), lo cual indica que enemigos naturales nativos se están adaptando a la plaga exótica. Los autores de este último trabajo han encontrado que varios productores de enemigos naturales iniciaron crías tanto de Necremnus artynes Walker (Hymenoptera: Eulophidae) como de Necremnus tidius Walker (Hymenoptera: Eulophidae) para liberarlos en el mercado mediterráneo en 2011. La distribución de N. artynes y N. tidius no está restringida al Mediterráneo. Según Van der Straten et al. (2011), otros enemigos naturales han sido reportados en experimentos realizados a campo abierto en Holanda. Después de exposición de diferentes estados de T. absoluta, se encontró que los ectoparasitoides Elachertus inunctus Nees (Eulophidae: Eulophidae) y Pnigalio soemius (Walker) (Hymenoptera: Eulophidae) (identificación por Christer Hansson, Lund University, Suecia) se desarrollaban bien en larvas jóvenes de la plaga. E. inunctus es un parasitoide 
de varias especies de microlepidópteros; Pnigalio soemius (Hymenoptera: Eulophidae) es un parasitoide generalista de muchas especies de microlepidópteros, dípteros y coleópteros. Además de los anteriores, esporádicamente se encontró Dicyphus errans (Wolff) (Heteroptera: Miridae), con alguna frecuencia en el verano, y Heterotoma sp. (Heteroptera: Miridae), muy ocasionalmente. Varias especies nativas de depredadores y parasitoides se han encontrado en el Mediterráneo alimentándose de T. absoluta (Al-Jboory et al., 2012). Zappalà et al. (2012) hicieron un levantamiento de parasitoides en el suroriente de Italia e identificaron trece especies que corresponden a diez géneros de las siguientes familias: Ichneumonidae, Braconidae, Eulophidae, Elasmidae, Pteromalidae y Trichogrammatidae. Posteriormente, Zappalà et al. (2013) reportan la existencia de varios depredadores y parasitoides atacando de manera espontánea a T. absoluta en cultivos de tomate en Europa y el norte de África. Muchos de ellos, principalmente Miridae nativos, han sido empleados en estrategias de manejo integrado de plagas (MIP) (Castane et al., 2011; Mollá et al., 2010; Zappalà et al., 2012; Chailleux et al., 2013). Sin embargo, aún se adelantan ensayos para identificar qué tan efectivas son estas especies de enemigos naturales en áreas invadidas por T. absoulta (Chailleux et al., 2012). Más de setenta especies de enemigos naturales generalistas han sido reportadas para $T$. absoluta en la región oeste del Paleoártico.

Colomo et al. (2002) y Luna et al. (2007, 2012), citados en Luna et al. (2015), reportan en Argentina un complejo de aproximadamente veinte especies de parasitoides para el control de T. absoluta. Además, refieren que tanto el endoparasitoide coinobionte Pseudapanteles dignus Muesebeck, 1938 (Hymenoptera: Braconidae) como el ectoparasitoide idiobionte Dineulophus phthorimaeae De Santis, 1983 (Hymenoptera: Eulophidae) alcanzaron hasta 50 $\%$ de parasitismo natural y presentaron atributos promisorios tanto para control biológico aumentativo como conservativo de T. absoluta. Estos parasitoides podrían ser aprovechados para su introducción en nuevas regiones infestadas por $T$. absoluta (Savino et al., 2012). Moya-Raygoza et al. (2012), citado en Luna et al. (2015), reportaron dos especies de parasitoides de huevos que ocurren naturalmente en cultivos de tomate en Argentina: Trichogramma pretiosum Riley, 1879 (Hymenoptera: Trichogrammatidae) y Encarsia porteri Mercet, 1928 (Hymenoptera: Aphelinidae). Los porcentajes de parasitismo fueron bajos $(<5 \%)$, pero cuando estos dos parasitoides de huevos coexisten, el $50 \%$ de los huevos de $T$. absoluta es parasitado por cualquiera de las dos especies, y no fue observado superparasitismo.

Luna et al. (2015) citan un estudio de Speranza et al. (2014) relacionado con un mapeo de cultivos de tomate en Argentina en los cuales un chinche nativo depredaba varios estados móviles. La especie fue identificada como Zelus obscuridorsis Stål (Hemiptera: Heteroptera: Reduviidae). Ensayos realizados para estimar la capacidad de depredar en varios estados de desarrollo de T. absoluta permitieron identificar que $Z$. obscuridorsis consumía larvas libres y adultos, pero no larvas que estuvieran dentro de minas, pupas o huevos.

Más de cuarenta especies de depredadores de T. absoluta ocurren en América del Sur, incluidas diferentes especies de Heteroptera (Hemiptera) y Debilia (Reduvidae) que depredan larvas de la plaga en Brasil (Desneux et al., 2010; Bueno et al., 2013). Entre los parasitoides reportados en Sudamérica para el control de T. absoluta cabe mencionar al Apanteles gelechiidivoris (Marchiori et al., 2004; Virgala 2006; Bajonero y Córdoba, 2007; Faria et al., 2008; Loni et al., 2011).

A. gelechiidivoris ha sido reportado en países sudamericanos tales como Colombia, Chile y Perú (Desneux et al., 2010; Urbaneja et al., 2013). En Colombia los estudios de este parasitoide han sido desarrollados en la Universidad Militar Nueva Granada, y dentro de estos se han destacado estudios de biología y ciclo reproductivo (Escobar et al., 2005; Bajonero et al., 2008), y de acción combinada entre dos tipos de control (Morales et al., 2014), entre otros.

\section{BIOECOLOGÍA DELPARASITOIDEApanteles gelechiidivoris}

Los huevos de A. gelechiidivoris tienen un promedio de 0,8 mm de largo. Se puede observar la presencia de más de un huevo dentro de la larva hospedera, lo que indica superparasitismo (Bajonero y Córdoba, 2007). En cuanto a su desarrollo larval se observa la existencia de tres estadios (Morales et al., 2014). Bajonero y Córdoba (2007) describen como característica del primer instar la prolongación del cuerpo en forma de gancho en la parte posterior del individuo. Asimismo, en proporción al cuerpo el tamaño de la cabeza es mucho mayor. En su segundo instar larval el cuerpo adquiere una mayor longitud y la cabeza corresponde al ancho del cuerpo. Durante el tercer instar la larva se alimenta de tejidos y contenido del hospedero y al final abandona el cuerpo de este. En la fase temprana de los estados de pupa pueden apreciarse dos manchas en la parte anterior, con una coloración amarillo pálido que se va oscureciendo a medida que avanza el desarrollo de la pupa hasta alcanzar una coloración negra. La mayoría de las veces la pupa presenta hilos blancos brillantes (Bajonero et al., 2008). Ambos sexos son de color oscuro y presentan dieciocho flagelómeros en las antenas, característica presente en los individuos que pertenecen a la subfamilia Microgastrinae (Campos, 2001). Las alas anteriores son hialinas y presentan una mancha oscura en la región estigmática. Existe una clara diferenciación entre sexos. Las hembras miden en promedio $4,12 \pm 0,17 \mathrm{~mm}$ y el


Los machos miden en promedio $4,08 \pm 0,12 \mathrm{~mm}$ y tienen un abdomen más angosto que el de las hembras (Bajonero et al., 2008). Una aproximación preliminar al potencial de control de este endoparasitoide ha sido obtenida mediante estudios de respuesta funcional. Bajonero et al. (2008) y Wanumen (2012) establecieron que el máximo número de hospederos de $A$. gelechiidivoris en condiciones de laboratorio es de 1 parasitoide : 12 hospederos a una temperatura en promedio de $20{ }^{\circ} \mathrm{C}$ y de 1 parasitoide : 6 hospederos en condiciones 
comerciales de invernadero a temperatura de $25^{\circ} \mathrm{C}$. En los dos casos se determinó que la respuesta funcional de $\mathrm{A}$. gelechiidivoris fue de tipo II.

\section{CONTROL QUÍMICO}

El método convencional de control en los sistemas de producción bajo invernadero y en campo abierto es la aplicación de insecticidas quimiosintéticos (Terzidis et al., 2014). Los ingredientes activos más utilizados para el control de plagas en los cultivos de tomate son tiocyclam, carbofuram, metamidofos, cypermetrina, metomil, imidacloprid y abamectina (Guedes et al., 1994). Sin embargo, algunos de estos insecticidas causan grandes desequilibrios en el medio ambiente (Medeiros et al., 2009).

Silva et al. (2016) evaluaron la resistencia de poblaciones de T. absoluta provenientes de cultivos comerciales de diferentes regiones de Brasil. Los autores reportan una correlación entre el uso excesivo del insecticida y la resistencia al producto. Sin embargo, uno de los mayores desafíos de la agricultura moderna es garantizar la selectividad de los agroquímicos de nueva generación.

\section{SELECTIVIDAD DE PRODUCTOS FITOSANITARIOS SOBRE} A. gelechiidivoris

Herrera (2013) determinó el efecto de varios productos fitosanitarios sobre A. gelechiidivoris. Estos productos provenían de diferentes orígenes de composición, tales como extractos vegetales, diversos tipos de biorracionales, entomopatógenos y productos de síntesis química. El insecticida spinosad, el hongo entomopatógeno B. bassiana y el fungicida penconazol tuvieron un efecto negativo sobre adultos de $A$. gelechiidivoris, mientras los extractos vegetales no presentaron efecto negativo sobre la mortalidad de adultos en condiciones de laboratorio. Cuando las evaluaciones se realizaron sobre pupas del parasitoide, todos los productos fueron considerados como inofensivos para A. gelechiidivoris, excepto el fungicida con ingrediente activo penconazol.

\section{CONCLUSIONES}

La distribución actual de T. absoluta en diferentes partes del mundo ha despertado el interés en realizar diversos estudios sobre estrategias potencialmente efectivas para su control. En Colombia los estudios adelantados sobre el controlador biológico nativo A. gelechiidivoris, presente en las principales zonas productoras de tomate bajo invernadero, indican que este parasitoide provee una oportunidad ideal para ser implementado en los programas de MIP. Sin embargo, la implantación en campo de este parasitoide debe hacerse bajo criterios adecuados ya que el uso de productos fitosanitarios no compatibles puede afectar su éxito como agente controlador. Así pues, es imprescindible adelantar estudios básicos de selectividad. No existe un método estandarizado para el caso del referido himenóptero, pero se podrían adaptar alguno de los métodos OILB existentes para otros parasitoides (Hassan, 1991, 1994) o adaptar el método de cálculo de la DL50 (Stark et al., 2007a, 2007b).

El mayor reto para los próximos años es la realización de estudios de efectos letales y subletales en condiciones más reales que el laboratorio (semicampo y campo), considerando además factores tales como la persistencia de los productos evaluados. Eso permitirá conocer la selectividad ecológica y planificar los tratamientos contra la plaga cuando los residuos ya no sean tóxicos para el enemigo natural.

El aumento del conocimiento en este campo contribuirá a la consolidación e intercambio de información en bases de datos como las propuestas en el pasado por Theiling $y$ Croft (1988) o las usadas actualmente por la OILB (Boller et al., 2005; OILB, 2017) o las casas comerciales (Biobest, 2017; Koppert, 2017; IPM Impact, 2017, entre otras).

\section{AGRADECIMIENTOS}

Los autores de este trabajo agradecen a la Vicerrectoría de Investigaciones de la Universidad Militar Nueva Granada por la financiación derivada de proyecto INV-CIAS-1783-Vigencia 2015.

\section{REFERENCIAS}

- Abbes K, Biondi A, Kurtulus A, Ricupero M, Russo A, Siscaro G y Zappalà L. 2015. Combined non-target effects of insecticide and high temperature on the parasitoid Bracon nigricans. PLOS ONE, 10(9):1-14.

- Agronet. 2016. Área cosechada, producción y rendimiento de tomate invernadero, 2013.

- Ajaya S, Ram P, Binu B, Pathour R y Naresh M. 2016. The first record of South American tomato leaf miner, Tuta absoluta (Meyrick 1917) (Lepidoptera: Gelechiidae) in Nepal. Journal of Entomology and Zoology Studies, 4(4):1359-1363.

- Al-Jboory I, Katbeh-Bader A y Al-Zaidi S. 2012. First observation and identification of some natural enemies collected from heavily infested tomato by Tuta absoluta (Meyrick) (Lepidoptera: Gelechiidae) in Jordan. Middle East Journal of Scientific Research, 11:435-438.

- Babendreier D, Bigler F y Kuhlmann U. 2005. Methods used to assess nontarget effects of invertebrate biological control agents of arthropod pests. Biocontrol, 50:821-870.

- Bajonero J y Córdoba N. 2007. Biología y ciclo reproductivo de Apanteles gelechiidivoris (Hymenoptera: Braconidae). Trabajo de grado, Facultad de Ciencias Básicas y Aplicadas, Universidad Militar Nueva Granada, Bogotá, 54 p.

- Bajonero J, Córdoba N, Cantor F, Rodríguez D y Cure JR. 2008. Biología y ciclo reproductivo de Apanteles gelechiidivoris (Hymenoptera: Braconidae), parasitoide de Tuta absoluta (Lepidoptera: Gelechiidae). Agronomía Colombiana, 26(3):417-426.

- Batalla-Carrera L, Morton A y García-del-Pino F. 2010. Efficacy of entomopathogenic nematodes against the tomato leafminer, Tuta absoluta, in laboratory and greenhouse conditions. Biocontrol, 55(4):523-530.

- Bawin T, De Backer L, Dujeu DL, Legrand P, Megido R, Francis F y Verheggen, F. 2014. Infestation level influences oviposition site selection in the tomato leafminer Tuta absoluta (Lepidoptera: Gelechiidae). Insects, 5(4):877-884.

- Biobest. 2017. Side-effect manual. http://www.biobestgroup.com/en/sideeffect-manual, consulta enero de 2017.

- Boller EF, Vogt H, Ternes P y Malavolta C. 2005. Working document on selectivity of pesticides. IOBC/WPRS Bulletin. https:/goo.gl/guvjEU, consulta septiembre de 2018.

- Bueno VHP, Van Lenteren JC, Lins Jr. JC, Calixto AM, Montes FC, Silva DB, Santiago LD y Pérez LM. 2013. New records of Tuta absoluta (Meyrick) (Lepidoptera: Gelechiidae) predation by Brazilian hemipteran predatory bugs. Journal of Applied Entomology, 137(1-2):29-34.

- Campos D. 2001. Avispas parasíticas de la familia Braconidae (Hymenoptera) en Colombia: diversidad genérica y distribución geográfica. Tesis de grado, Facultad de Ciencias, Universidad Nacional de Colombia, 60 p. 
- Campos MR, Rodrigues ARS, Silva WM, Silva TBM, Silva VRF, Guedes RNC y Siqueira HA. 2014. Spinosad and the tomato borer Tuta absoluta: A bioinsecticide, an invasive pest threat, and high insecticide resistance. PLOS ONE, 9(8):e103235.

- Caparros MR, Haubruge E y Verheggen F. 2012. First evidence of deuterotokous parthenogenesis in the tomato leaf-miner, Tuta absoluta (Meyrick) (Lepidoptera: Gelechiidae). Journal of Pest Science, 85(4):409-412.

- Caparros MR, Haubruge E y Verheggen F. 2013. Pheromone-based management strategies to control the tomato leafminer, Tuta absoluta (Lepidoptera: Gelechiidae): A review. Biotechnologie, Agronomie, Société et Environnement= Biotechnology, Agronomy, Society and Environment, 17(3):475-482

- Castañé C, Arnó J, Gabarra R y Alomar O. 2011. Plant damage to vegetable crops by zoophytophagous mirid predators. Biological Control, 59(1):22-29.

- Castiglioni E, Giani G, Binnewies C y Bentancur O. 2008. Susceptibilidad de la chinche Piezodorus guildinii Westwood (Hemiptera: Pentatomidae) al insecticida Endosulfán. Agrociencia, 12:31-34.

- Chailleux A, Desneux N, Seguret N, Do Thi Khanh H, Maignet P y Tabone E. 2012. Assessing European egg parasitoids as a mean of controlling the invasive South American tomato pinworm Tuta absoluta. PLOS ONE, 7:e48068

- Chailleux A, Bearez P, Pizzol J, Amiens-Desneux E, Ramirez-Romero R y Desneux N. 2013. Potential for combined use of parasitoids and generalist predators for biological control of the key invasive tomato pest Tuta absoluta. Journal of Pest Science, 86(3):541-553.

- Chermiti B y Abbes K. 2012. Comparison of pheromone lures used in mass trapping to control the tomato leafminer Tuta absoluta (Meyrick, 1917) in industrial tomato crops in Kairouan (Tunisia). EPPO Bull, 42:241-248.

- Cocco A, Deliperi S y Delrio G. 2013. Control of Tuta absoluta (Meyrick) (Lepidoptera: Gelechiidae) in greenhouse tomato crops using the mating disruption technique. Journal of Applied Entomology, 137:16-28.

- Cocco A, Serra G, Lentini A, Deliperi S y Delrio G. 2014. Spatial distribution and sequential sampling plans for Tuta absoluta (Lepidoptera: Gelechiidae) in greenhouse tomato crops. Pest Management Science, 71(9):1311-1323.

- Collavino MD y Giménez RA. 2001. Efecto del Imidacloprid en el control de la polilla del tomate Tuta absoluta (Meyrick). Idesia Arica, 26:65-72.

- Colomo MV, Berta DC y Chocobar MJ. 2002. El complejo de himenópteros parasitoides que atacan a la polilla del tomate Tuta absoluta (Lepidoptera: Gelechiidae) en la Argentina. Acta Zoologica Lilloana, 46(1):81-92.

- Delbene JA. 2003. Evaluación de cepas nativas de los hongos entomopatógenos Beauveria sp. y Metarhizium sp sobre el control de polilla del tomate Tuta absoluta Meyrick. Pontificia Universidad Católica de Valparaíso, $41 \mathrm{p}$.

- Denez MD, Mello D, Silva DA y Moraes MA. 2014. Insecticide selectivity for Doru lineare (Dermaptera: Forficulidae). Revista Brasileira de Milho e Sorgo, 13:107-115.

- Desneux N, Decourtye A y Delpuech J-M. 2007. The sublethal effects of pesticides on beneficial arthropods. Annual Review of Entomology, 52:81-106.

- Desneux N, Wajnberg E, Wyckhuys KG, Burgio G, Arpaia S, Narváez-Vasquez C ... y Urbaneja A. 2010. Biological invasion of European tomato crops by Tuta absoluta: Ecology, geographic expansion and prospects for biological control. Journal of Pest Science, 83(3):197-215

- EPPO Global Database. 2017. Tuta absoluta (GNORAB). Distribution. https:// gd.eppo.int/taxon/GNORAB/distribution, consulta septiembre de 2018.

- Escobar A, Cantor F y Cure J. 2005. Contribución al conocimiento de la biología de Apanteles sp. (Hymenoptera: Braconidae). Revista Facultad de Ciencias Básicas, 1:125-126.

- Faria CA, Torres JB, Fernandes AMV y Farias AMI. 2008. Parasitism of Tuta absoluta in tomato plants by Trichogramma pretiosum Riley in response to host density and plant structures. Ciência Rural, 38:1504-1509.

- Garcia P. 2009. Sublethal effects of pyrethroids on insect parasitoids: What we need to further know. IntechOpen. https://goo.gl/uygjN7, consulta septiembre de 2018 .

- Ghoneim K. 2014. Parasitic insects and mites as potential biocontrol agents for a devastative pest of tomato Tuta absoluta Meyrick (Lepidoptera: Gelechiidae) in the world: A review. International Journal of Advanced Research, 2:81-115.

- Giustolin TA, Vendramim JD, Alves SB y Vieira SA, 2001. Pathogenicity of Beauveria bassiana (Bals.) Vuill. to Tuta absoluta (Meyrick) (Lepidoptera: Gelechiidae) reared on two genotypes of tomato. Neotropical Entomology, 30(3):417-421.

- Gómez J. 2014. Desarrollo de un bioplaguicida a base de granulovirus para el control de Tuta absoluta (Meiryck, 1917) (Lepidoptera: Gelechiidae) en el cultivo de tomate de mesa Solanum lycopersicum L. bajo cubierta. Tesis de doctorado, Facultad de Agronomía, Universidad Nacional de Colombia, Bogotá, $216 \mathrm{p}$

- Gómez-Valderrama J, Herrera L, Uribe-Vélez D, López-Ferber M y Villamizar L. 2014. An immunological method for granulovirus detection in larvae of Tuta absoluta: Searching for isolates with prospects for biological control of this pest in Colombia. International Journal of Pest Management, 60(2):136-143.

- González A, Altesor P, Sellanes C y Rossini C. 2012. Aplicación de feromonas sexuales en el manejo de lepidópteros plaga de cultivos agrícolas (pp. 343360). En: Rojas JC y Malo EA (Eds.). Temas selectos en ecología química de insectos, El Colegio de la Frontera Sur, Tapachula, Chiapas.

- González-Cabrera J, Mollá O, Montón H y Urbaneja A. 2010. Efficacy of Bacillus thuringiensis (Berliner) in controlling the tomato borer, Tuta absoluta (Meyrick) (Lepidoptera: Gelechiidae). Biocontrol, 56:71-80.

- Grousset F, Suffert M y Petter F. 2015. EPPO Study on pest risks associated with the import of tomato fruit. EPPO Bulletin, 45:153-156.

- Guedes RNC y Picanço MC. 2012. The tomato borer Tuta absoluta in South America: Pest status, management and insecticide resistance. EPPO Bulletin, 42:211-216.

- Guedes RNC, Picanço MC, Matioli AL y Rocha DM. 1994. Effect of insecticide and tomato cultivation systems in the control of Scrobipalpuloides absoluta (Meyrick) (Lepidoptera: Gelechiidae). Anais da Sociedade Entomológica do Brasil, 23:321-325.

- Gul A, Mahmood I, Imadi SR y Shazadi K. 2016. Effects of pesticides on environment (pp. 253-269). En: Hakeem KR, Akhtar MS y Akmar SN (Eds.). Plant, soil and microbes, Vol. 1. Springer, Cham.

- Hardy M. 2011. Using selective insecticides in sustainable IPM. CAB Reviews: Perspectives in Agriculture, Veterinary Science, Nutrition and Natural Resources, 6(39). https://goo.gl/w1ongL, consulta septiembre de 2018.

- Hassan MN y Al-Zaidi S. 2009. Tuta absoluta: A serious pest advancing in the Mediterranean region. Role of pheromones in management strategies. Integrated Pest Management, 51:85-87.

- Hassan SA, Bigler F, Bogenschütz H, Boller E, Brun J, Calis JNM y Mansour F. 1991. Results of the fifth joint pesticide testing programme carried out by the IOBC/WPRS-Working Group "Pesticides and Beneficial Organisms". Entomophaga, 36(1):55-67.

- Hassan SA, Bigler F, Bogenschütz H, Boller E, Brun J, Calis JNM, CoremansPelseneer J, Duso C, Grove A, Heimbch U, Helyer N, Hokannen H, Lewis GB, Mansour F, Moreth L, Polgar L, Samsoe-Petersen L, Sauphanor B, Stäubli A, Sterk G, Vainio A, Veire MV, Viggiani G y Vogt H. 1994. Results of the sixth joint pesticide testing programme of the IOBC/WPRS. Entomophaga, 39(1):107-119.

- Herrera M. 2013. Determinación del efecto de productos fitosanitarios utilizados en el cultivo del tomate sobre el parasitoide Apanteles gelechiidivoris Marsh (Hymenoptera: Braconidae). Trabajo de grado, Facultad de Ciencias Básicas y Aplicadas, Universidad Militar Nueva Granada, Bogotá, 64 p.

- IPM Impact. 2017. Side-effects database. http://www.ipmimpact.com/, consulta enero de 2017.

- Jaramillo JE, Sánchez GD, Rodríguez VP, Aguila PA, Gil LF, Hío JC, Pinzón LM, García MC, Quevedo D, Zapata MÁ, Restrepo JF y Guzmán M. 2013. Tecnología para el cultivo de tomate bajo condiciones protegidas. Corpoica, Bogotá, 482 p.

- Jeschke P. 2016. Propesticides and their use as agrochemicals. Pest Management Science, 72:210-225.

- Kaoud HA. 2014. Alternative methods for the control of Tuta absoluta. GJMAS Journal, 2:41-46

- Koppert. 2017. Efectos secundarios. https://www.koppert.es/efectossecundarios/, consulta enero de 2017.

- Lobos E, Occhionero M, Werenitzky D, Fernandez J, Gonzalez LM, Rodriguez C y Oehlschlager AC. 2013. Optimization of a trap for Tuta absoluta Meyrick (Lepidoptera: Gelechiidae) and trials to determine the effectiveness of mass trapping. Neotropical Entomology, 42(5):448-457.

- Loni A, Rossi E y Van Achterberg K. 2011. First report of Agathis fuscipennis in Europe as parasitoid of the tomato leafminer Tuta absoluta. Bulletin of Insectology, 64:115-117.

- Luna MG, Sánchez NE y Pereyra PC. 2007. Parasitism of Tuta absoluta (Lepidoptera: Gelechiidae) by Pseudapanteles dignus (Hymenoptera: Braconidae) under laboratory conditions. Environmental Entomology, 36:887-893.

- Luna M, Pereyra P y Sánchez N. 2012. Biological control of Tuta absoluta (Lepidoptera: Gelechiidae) in protected tomato crops in Argentina. IOBCWPRS Bulletin, 80:177-182. 
- Luna M, Pereyra P, Coviella C, Nieves E, Savino V, Salas-Gervassio N, Luft E, Virla E y Sánchez N. 2015. Potential of biological control agents against Tuta absoluta (Lepidoptera: Gelechiidae): Current knowledge in Argentina. Florida Entomologist, 98(2):489-494.

- Mafra-Neto A, Borges R, Stoltman L y Zeni D. 2010. Pheromone tools for early detection and control of the invasive tomato leafminer, Tuta absoluta. Entomological Society of America 2010. 58th Annual Meeting, diciembre 1215, San Diego, California.

- Marchiori CH, Silva CG y Lobo P. 2004. Parasitoids of Tuta absoluta (Meyrick, 1917) (Lepidoptera: Gelechiidae) collected on tomato plants in lavras, State of Minas Gerais, Brazil. Revista Brasileira de Biología, 64:551-552.

- Martins JC, Picanço MC, Bacci L, Liz RS y Morais HC. 2016. Life table determination of thermal requirements of the tomato borer Tuta absoluta. Journal of Pest Science, 89(4):897-908.

- Medeiros MA. 2007. Papel da biodiversidade no manejo da traça-do-tomateiro Tuta absoluta (Meyrick, 1917) (Lepidoptera: Gelechiidae). Revista Brasileira de Agroecologia, 4(1). https://goo.gl/zhv3GP, consulta septiembre de 2018.

- Medeiros MA, Sujii ER, Rasi GC, Liz RS y Morais HC. 2009. Padrão de oviposição e tabela de vida da traça-do-tomateiro Tuta absoluta (Meyrick) (Lepidoptera, Gelechiidae). Revista Brasileira de Entomologia 53:452-456.

- Megido RC, Haubruge Ey Verheggen FJ. 2013. Pheromone-based management strategies to control the tomato leafminer, Tuta absoluta (Lepidoptera: Gelechiidae). A review. Revue de Biotechnologie, Agronomie, Société et Environnement, 17:475-482.

- Michereff Filho M, Vilela E, Gulab N, Attygalle A, Svatos A y Meinwaldf J. 2000. Initial studies of mating disruption of the tomato moth. Journal of the Brazilian Chemical Society, 11:621-628.

- Mochizuki F, Miyake Y, Ishibashi N y Fukumoto T. 2012. Method for disrupting mating of Tuta absoluta. European Patent application. EP 2465349 A2. ShinEtsu Chemical Co. Ltd. Tokio: European Patent Application.

- Mohan M, Sushil SN, Bhatt JC, Gujar GT y Gupta HS. 2007. Synergistic interaction between sublethal doses of Bacillus thuringiensis and Campoletis chlorideae in managing Helicoverpa armigera. BioControl, 53:375-386.

- Mollá O, Alonso M, Monton H, Beitia F, Verdu M, González-Cabrera J y Urbaneja A. 2010. Control biológico de Tuta absoluta. Catalogación de enemigos naturales y potencial de los míridos depredadores como agentes de control. Phytoma Spain, 217:42-46.

- Monserrat A. 2009. La polilla del tomate Tuta absoluta en la región de Murcia: bases para su control. Serie Técnica y de Estudios, 34:74.

- Morales J, Rodríguez D y Cantor F. 2013. Estandarización de la cría masiva de Apanteles gelechiidivoris Marsh (Hymenoptera: Braconidae) para el control de Tuta absoluta Meyrick (Lepidoptera: Gelechiidae). Revista Facultad de Ciencias Básicas, 9:20-37.

- Morales J, Muñoz L, Rodríguez D y Cantor F. 2014. Acción combinada de feromona sexual y de avispas Apanteles gelechiidivoris para el control de Tuta absoluta en cultivos de tomate. Acta Biológica Colombiana, 19:175-184.

- Moya-Raygoza G, Virla E y Luft E. 2012. Diversity of egg parasitoids attacking Dalbulus maidis (Hemiptera: Cicadellidae) populations at low and high elevation sites in Mexico and Argentina. Florida Entomologist, 95:105-112.

- OILB, 2017. Database on selectivity of pesticides. Table: http://www.iobcwprs. org/ip_ipm/03022_IOBC_PesticideDatabase_2005.pdf. Explanations: http:// www.iobcwprs.org/ip_ipm/03021_IOBC_WorkingDocumentPesticides_ Explanations.pdf, consulta enero de 2017.

- Pires LM, Marques EJ, De Oliveira JV y Alves SB. 2010. Seleção de isolados de fungos entomopatogênicos para o controle de Tuta absoluta Meyrick (Lepidoptera: Gelechiidae) e sua compatibilidade com alguns inseticidas usados na cultura do tomateiro. Biological Control, 39:977-984.

- Ramírez L, Ramírez N, Fuentes LS, Jiménez J y Hernández Fernández J. 2010. Estandarización de un bioensayo y evaluación preliminar de tres formulaciones comerciales de Bacillus thuringiensis sobre Tuta absoluta Meyrick (Lepidoptera: Gelechiidae). Revista Colombiana de Biotecnología, 12:12-21.

- Razuri V y Vargas E. 1975. Biology and behaviour of Scrobipalpula absoluta Meyrick (Lep., Gelechiidae) on tomatoes. Revista Peruana de Entomología, 18:84-89.

- Roda AL, Brambila J, Barria J, Euceda X y Korytkowski C. 2015. Efficiency of trapping systems for detecting Tuta absoluta (Lepidoptera: Gelechiidae). Journal of Economic Entomology, 108:2648-2654.

- Rodríguez M, Gerding M y France A. 2006. Efectividad de aislamientos de hongos entomopatógenos sobre larvas de polilla de tomate Tuta absoluta Meyrick (Lepidoptera: Gelechiidae). Agricultura Técnica, 66(2):159-165.
- Roubos CR, Rodriguez-Saona C e Isaacs R. 2014. Mitigating the effects of insecticides on arthropod biological control at field and landscape scales. Biological Control, 75:28-38.

- Santos MF, Campos MR, Bravim JN, Bravim J, Oliveira EE y Guedes RNC. 2016. Non-targeted insecticidal stress on the neotropical brown stink bug Euschistus heros. Crop Protection, 82:10-16.

- Sattar S, Saljoqi A, Arif M y Sattar H. 2011. Toxicity of some new insecticides against Trichogramma chilonis (Hymenoptera: Trichogrammatidae) under laboratory and extended laboratory conditions. Pakistan Journal of Zoology, 43(6):1117-1125.

- Savino V, Coviella CE y Luna MG. 2012. Reproductive biology and functional response of Dineulophus phtorimeae, a natural enemy of the tomato moth, Tuta absoluta. Journal of Insect Science, 12:1-14.

- Shashank P, Chandrashekahr K, Meshram N y Shreedevi K. 2015. Occurrence of Tuta absoluta (Lepidoptera: Gelechiidae) an invasive pest from India. Indian Journal of Entomology, 77(4):323-329.

- Silva TBM, Silva WM, Campos MR, Silva JE, Ribeiro LMS y Siqueira HAA. 2016. Susceptibility levels of Tuta absoluta (Meyrick) (Lepidoptera: Gelechiidae) to minor classes of insecticides in Brazil. Crop Protection, 79:80-86.

- Siqueira H, Guedes RNC, Fragoso DB y Magalhaes LC. 2001. Abamectin resistance and synergism in Brazilian populations of Tuta absoluta (Meyrick) (Lepidoptera: Gelechiidae). International Journal of Pest Management, 47:247-251.

- Speranza S, Melo M, Luna M y Virla E. 2014. First record of Zelus obscuridorsis (Hemiptera:Reduviidae) as a predator of the South American tomato leafminer Tuta absoluta (Lepidoptera: Gelechiidae). Florida Entomologist, 97:295-297.

- Stark JD, Vargas R y Banks JE. 2007a. Incorporating ecologically relevant measures of pesticide effect for estimating the compatibility of pesticides and biocontrol agents. Journal Economic Entomology, 100(4):1027-1032.

- Stark JD, Sugayama RL y Kovaleski A. 2007b. Why demographic and modeling approaches should be adopted for estimating the effects of pesticides on biocontrol agents. BioControl, 52(3):365-374.

- Taha AM, Afsah AFE y Fargalla FH. 2013. Evaluation of the effect of integrated control of tomato leafminer Tuta absoluta with sex pheromone and insecticides. Nature and Science, 11(7):26-29.

- Talebi K, Kavousi A y Sabahi Q. 2008. Impacts of pesticides on arthropod biological control agents. Pest Technology, 2:87-97.

- Terzidis AN, Wilcockson S y Leifert C. 2014. The tomato leaf miner Tuta absoluta: Conventional pest problem, organic management solutions? Organic Agriculture, 4:43-61.

- Theiling KM y Croft BA. 1988. Pesticide side-effects on arthropod natural enemies: A database summary. Agriculture, Ecosystems and Environment, 21:191-218.

- Tonnang H, Mohamed S, Khamis F y Ekesi S. 2015. Identification and risk assessment for worldwide invasion and spread of Tuta absoluta with a focus on Sub-Saharan Africa: Implications for phytosanitary measures and management. PLOS ONE, 10(8): e0135283.

- Torres Gregorio J, Argente J, Díaz MA y Yuste A. 2009. Aplicación de Beauveria bassiana en la lucha biológica contra Tuta absoluta. Agrıcola Vergel: Fruticultura, Horticultura, Floricultura, 326:129-132.

- Tropea Garzia G, Siscaro G, Biondi A y Zappalà L. 2012. Tuta absoluta a South American pest of tomato now in the EPPO region: Biology, distribution and damage. EPPO Bulletin, 42:205-210.

- Umoru P y Powell W. 2002. Sub-lethal effects of the insecticides pirimicarb and dimethoate on the aphid parasitoid Diaeretiella rapae (Hymenoptera: Braconidae) when attacking and developing in insecticide-resistant hosts. Biocontrol Science and Technology, 12:605-614.

- Urbaneja A, Montón y Molla O. 2009. Suitability of the tomato borer Tuta absoluta as prey for Macrolophus pygmaeus and Nesidiocoris tenuis. Journal of Applied Entomology, 133(2):22-26.

- Urbaneja A, Desneux N, Gabarra R, Arnó J, González-Cabrera J, Mafra Neto A, Stoltman L, Pinto A y Parra JRP. 2013. Biology, ecology and management of the South American tomato pinworm, Tuta absoluta. Potential invasive pests of agricultural crops (pp. 98-125). En: Peña J. Potential Invasive Pests of Agricultural Crops. CAB International, Washington, D. C.

- Vacas S, Alfaro C, Primo J y Navarro-Llopis V. 2011. Studies on the development of a mating disruption system to control the tomato leafminer, Tuta absoluta Povolny (Lepidoptera: Gelechiidae). Pest Management Science, 67(11):1473-1480.

- Vacas S, López J, Primo J y Navarro-Llopis V. 2013. Response of Tuta absoluta (Lepidoptera: Gelechiidae) to different pheromone emission levels in greenhouse tomato crops. Environmental Entomology, 42:1061-1068. 
- Vallejo FA. 1999. Mejoramiento genético y producción de tomate en Colombia. Universidad Nacional de Colombia, Palmira.

- Van der Straten M, Potting R y Van der Linden A. 2011. Introduction of the tomato leafminer Tuta absoluta into Europe. En: Proceedings of the Netherlands Entomological Society Meeting, 22:23-30.

- Vargas H. 1970. Observaciones sobre la biología y enemigos naturales de la polilla del tomate, Gnorimoschema absoluta (Meyrick) (Lep: Gelechiidae). Idesia, 1:75-110.

- Vélez R. 1997. Plagas agrícolas de impacto económico en Colombia: bionomía y manejo integrado. Universidad de Antioquia, Medellín.

- Virgala R. 2006. Evaluación de algunos insecticidas para el control de la "polilla del tomate", Tuta absoluta (Lepidoptera: Gelechiidae) y su efecto residual sobre el parasitoide Trichogrammatoidea bactrae (Hymenoptera: Trichogrammatidae). Revista de la Sociedad Entomológica Argentina, 65:57-65.

- Wanumen A. 2012. Evaluación de diferentes densidades de liberación de Apanteles gelechiidivoris Marsh (Hymenoptera: Braconidae) para el control de Tuta absoluta Meyrick (Lepidoptera: Gelechiidae) en condiciones comerciales. Tesis de maestría, Facultad de Ciencias Básicas y Aplicadas, Universidad Militar Nueva Granada, Bogotá, 62 p.
- Zappalà L, Bernardo U, Biondi A, Cocco A, Deliperi S, Delrio G, Giorgini M, Pedata P, Rapisarda C, Garzia GT y Siscaro G. 2012. Recruitment of native parasitoids by the exotic pest Tuta absoluta in Southern Italy. Bulletin of Insectology, 65:51-61.

- Zappalà L, Biondi A, Alma A, Ibrahim, Al-Jboory IJ, Arnò J, Bayram A, Chailleux A, El-Arnaouty A, Gerling D, Guenaoui Y, Shaltiel-Harpaz L, Siscaro G, Stavrinides M, Tavella L, Vercher R, Urbaneja A y Desneux N. 2013. Natural enemies of the South American moth, Tuta absoluta, in Europe, North Africa and Middle East, and their potential use in pest control strategies. Journal of Pest Science, 86(4):635-647. 